\title{
Human Health and Medical Engineering
}

The 2014 International Conference on Human Health and Medical Engineering (HHME 2014) was held in The Ramada Plaza Optics Valley Hotel Wuhan from December 7, 2014 in Wuhan, China. This conference was sponsored by the Information Technology and Industrial Engineering Research Center.

HHME 2014 was a forum for presentation of new research results and demonstration of new systems and techniques in the broad field of human health and medical engineering. The forum aimed to bring together researchers, developers, and users from around the world for sharing state-of-art results, for exploring new areas of research and development, and to discuss emerging issues facing human health and medical engineering.

For this conference, we received more than 200 submissions from 5 countries and regions. Each of the papers was reviewed carefully by two famous specialists in this field. After receiving the revised papers on the basis of the specialists' advice, finally we selected 27 papers for this special issue of Technology and Health Care. In addition to the regular publications of the papers, we also invited several specialists and scholars in this field to make keynote speeches at the conference. We would like to take this opportunity to express our thanks to the individuals and organizations for their efforts to serve the conference.

We would like to extend our thanks to members of academic committee for their effort to the conference; especially, we would like to thank to members of organizing committee for their hard work; finally, we would like to express our appreciation to all the participants of this conference.

Zhen-Yu Du Guest Editor 